Gut and Liver, Vol. 10, No. 4, July 2016, pp. 595-603

\title{
Disease Phenotype, Activity and Clinical Course Prediction Based on C-Reactive Protein Levels at Diagnosis in Patients with Crohn's Disease: Results from the CONNECT Study
}

Jee Hye Kwon ${ }^{1}$, Jong Pil Im¹, Byong Duk Ye², Jae Hee Cheon ${ }^{3}$, Hyun Joo Jang ${ }^{4}$, Kang Moon Lee ${ }^{5}$, You Sun Kim ${ }^{6}$, Sang Wook Kim ${ }^{7}$, Young Ho Kim ${ }^{8}$, Geun Am Song ${ }^{9}$, Dong Soo $\mathrm{Han}^{10}$, Won Ho Kim ${ }^{3}$, and Joo Sung Kim ${ }^{1}$

${ }^{1}$ Department of Internal Medicine and Liver Research Institute, Seoul National University College of Medicine, ${ }^{2}$ Department of Internal Medicine, Asan Medical Center, University of Ulsan College of Medicine, ${ }^{3}$ Department of Internal Medicine and Institute of Gastroenterology, Yonsei University College of Medicine, Seoul, ${ }^{4}$ Department of Internal Medicine, Hallym University Dongtan Sacred Heart Hospital, Hwaseong, ${ }^{5}$ Department of Internal Medicine, St. Vincent's Hospital, The Catholic University of Korea College of Medicine, Suwon, ${ }^{6}$ Department of Internal Medicine, Inje University College of Medicine, Seoul, ${ }^{7}$ Department of Internal Medicine, Chonbuk National University Medical School, Jeonju, ${ }^{8}$ Department of Internal Medicine, Sungkyunkwan University School of Medicine, Seoul, ${ }^{9}$ Department of Internal Medicine, Pusan National University School of Medicine, Busan, and ${ }^{10}$ Department of Internal Medicine, Hanyang University Guri Hospital, Guri, Korea

Background/Aims: C-reactive protein (CRP) is an easily measured index of disease activity, but its ability to predict clinical course is controversial. We therefore designed a study to determine whether the CRP level at Crohn's disease (CD) diagnosis is a valuable indicator of the disease phenotype, activity, and clinical course. Methods: We retrospectively analyzed $705 \mathrm{CD}$ patients from 32 institutions. The patients were classified into two groups according to CRP level. The patients' demographic and clinical characteristics and their use of immunosuppressive or biological agents were recorded. Disease location and behavior, hospitalization, and surgery were analyzed. Results: A high CRP was associated with younger age, steroid use, colonic or ileocolonic location, high CD activity index, and active inflammation at colonoscopy $(p<0.001)$. As the disease progressed, patients with high CRP were more likely to exhibit strictures $(p=0.027)$. There were significant differences in the use of 5-aminosalicylic acid, antibiotics, corticosteroids, azathioprine, and infliximab $(p<0.001, p<0.001, p<0.001, p<0.001$, and $p=0.023$, respectively). Hospitalization was also more frequent in patients with high CRP. Conclusions: The CRP level at diagnosis is useful for evaluating the phenotype, activity, and clinical course of CD. Closer follow-up strategies, with early aggressive treatment, could be considered for patients with high CRP. (Gut Liver 2016;10:595-603)

Key Words: Crohn disease; C-reactive protein; Clinical course

\section{INTRODUCTION}

The clinical manifestation of Crohn's disease (CD) is heterogeneous, and the natural course is unpredictable, with phenotypic changes over time..$^{1-5}$ The development of prognostic markers would enable early treatment for severe CD. Although several clinical indices and serological and fecal markers, such as calprotectin, and genetic biomarkers, such as nucleotide-binding oligomerization domain-containing protein 2 , have been evaluated for the prediction of clinical course, a definitive predictive marker has not been fully established. ${ }^{6-10}$

C-reactive protein (CRP) is a surrogate marker of acute inflammation induced by infection, stress, tissue necrosis, trauma, and malignancies. ${ }^{11}$ CRP is synthesized principally by hepatocytes in response to stimulation by interleukin-1, interleukin-6 (IL-6), and tumor necrosis factor- $\alpha$ (TNF- $\alpha){ }^{12}$ Recent studies have suggested that CRP is also produced by peripheral lymphocytes, inflamed kidneys, alveolar macrophage, and adipocytes in quantities insufficient to affect plasma CRP levels. ${ }^{13,14}$ CRP functions independent of physiological and pathological conditions and plasma CRP levels in patients with CD. ${ }^{11}$ Therefore, CRP production by the liver may be the only factor determining the plasma CRP level, suggesting the potential of CRP as a valuable marker to diagnose and monitor CD patients.

Estimating CRP may be useful to evaluate disease activity because it requires an inexpensive and easily accessible test; in addition, compared to other inflammatory markers, CRP has a

\footnotetext{
Correspondence to: Jong Pil Im

Department of Internal Medicine and Liver Research Institute, Seoul National University College of Medicine, 101 Daehak-ro, Jongno-gu, Seoul 03080, Korea
}

Tel: +82-2-2072-0638, Fax: +82-2-743-6701, E-mail: jpim0911@snu.ac.kr

Received on August 24, 2015. Revised on October 2, 2015. Accepted on October 19, 2015. Published online March 30,2016

pISSN 1976-2283 eISSN 2005-1212 http://dx.doi.org/10.5009/gnl15411

@) This is an Open Access article distributed under the terms of the Creative Commons Attribution Non-Commercial License (http://creativecommons.org/licenses/by-nc/4.0) which permits unrestricted non-commercial use, distribution, and reproduction in any medium, provided the original work is properly cited. 
short half-life of 19 hours. ${ }^{15}$ Several studies have demonstrated that CRP levels are associated with the clinical, radiographic, endoscopic, and histological activity in CD. ${ }^{16-18}$ However, whether CRP levels can predict the clinical course is not known. Higher CRP levels at the time of diagnosis or relapse have been associated with more frequent and severe short- and medium-term relapses. ${ }^{11,19}$ A retrospective study from Portugal reported that high CRP in CD predicts nonresponse to infliximab. ${ }^{20}$ However, these data were from a single-center study with a small number of subjects, thus limiting the generalizability of the results. There is lack of reports that provide conclusive evidence for predicting aggressive clinical course and establishing treatment plan.

We therefore conducted a multicenter cohort study of a large number of $\mathrm{CD}$ subjects to determine if the CRP level at diagnosis is a valuable predictive marker of disease phenotype, activity, and clinical course.

\section{MATERIALS AND METHODS}

\section{Study population}

We reviewed a retrospective clinical database that included patients diagnosed with CD between July 1982 and December 2008 who were enrolled in the Crohn's Disease Clinical Network and Cohort (CONNECT) study. The gastroenterology clinics of 32 institutions in Korea participated in this cohort study, which was performed by the Korean Association for the Study of Intestinal Diseases. ${ }^{21,22}$ The institutions comprised 30 university hospitals and two community hospitals. Patients were eligible for the study if they had a measurable CRP level at the time of diagnosis, they did not have coexisting infectious colitis, they were followed up for longer than 6 months (to identify longterm clinical outcomes), and the status of their CD was evaluated before enrollment and at the end of the study. We excluded patients with incomplete clinical data $(n=100)$, those for whom CRP level at diagnosis was unavailable $(n=490)$, and those with lack of medical record from the referral hospitals $(n=79)$, and those who were followed up for less than 6 months $(n=8)$.

Patients were classified into two groups based on CRP $>20$ $\mathrm{mg} / \mathrm{L}$ and $\leq 20 \mathrm{mg} / \mathrm{L}$.

The diagnosis of CD was confirmed in all cases by previously established guidelines based on clinical history, endoscopic and radiological examinations, and histopathological findings. ${ }^{23}$

\section{Data collection and outcome measurement}

Clinical data at initial presentation of $\mathrm{CD}$ and subsequent follow-up visits were retrieved for analysis from the electronic medical record system. The collected data included age, gender, length of follow-up, disease location, and behavior at the time of diagnosis according to the Montreal classification. Disease location at final follow-up was categorized as colorectum, jejunum, ileum, or esophagogastroduodenum. The development of intestinal strictures, perianal fistula, and other fistulas and the time required to develop these lesions were assessed. Other fistulas were defined as abnormal tracks formed between the gut and gut, skin, an abscess cavity, or other hollow structures including the bladder, urethra, uterus, or vagina. We also recorded complications including abscesses, perforations, intestinal cancers, and primary sclerosing cholangitis.

Clinical disease activity at the time of diagnosis was assessed using the Crohn's disease activity index (CDAI), with a score of $>150$ indicating active disease. Among the typical endoscopic features of $\mathrm{CD}$, a cobblestone appearance and longitudinal ulcers were reviewed because these lesions can indicate increased CD activity. We recorded medication use during follow-up period, including 5-aminosalicylic acid (5-ASA), antibiotics, budesonide, and corticosteroids. The use of immunosuppressants, including azathioprine, 6-mercaptopurine, methotrexate, and TNF blockers such as infliximab and adalimumab, was also reviewed. Time from CD diagnosis to first use of azathioprine or TNF blockers was calculated to assess the length of time before these drugs were required to treat severe disease. Disease-related hospitalization (including readmissions) and surgery data were assessed because these can indicate a more severe course in $\mathrm{CD}^{24}$ The collected data regarding hospitalization included the total number of hospital admissions and the readmission rate. In this analysis, readmission was defined as two or more admission of a discharged patient for further inpatient care as a new episode.

The proportion of patients who underwent surgery was recorded, and additional data including the total number of surgeries, resurgeries, and survival rates were obtained. These patients were analyzed according to the causative lesion for surgery or resurgery: intestinal or perianal lesion. Resurgery was defined as surgery at the same lesion site or at another site for a relapsed or uncontrolled lesion or to repair temporary structures from a previous surgery such as ileostomy repair. This study protocol was reviewed and approved by the Institutional Review Board of each participating medical center.

\section{Statistical analysis}

Continuous variables are presented as means \pm standard deviation when appropriate. Categorical variables were summarized as frequency and percentage. We performed univariate analyses using an independent t-test for continuous variables and the Pearson chi-square test, Fischer exact test, or linear by linear association for categorical variables. Kaplan-Meier method and log-rank test were used to determine the cumulative probability of stricture, perianal fistula, other fistula, use of azathioprine and TNF blocker, and risk of surgery according to the CRP level at diagnosis. Binary logistic regression analysis was performed using a forward stepwise selection method to evaluate risk factors affecting hospital admission of patients with CD. A multivariate analysis was performed if the univariate analysis yielded 
$\mathrm{p}<0.25$. A p-value $<0.05$ was considered to indicate statistical significance. All analyses were performed using IBM SPSS version 20.0 (IBM Corp., Armonk, NY, USA).

\section{RESULTS}

\section{Demographic and clinical characteristics of patients}

A total of $705 \mathrm{CD}$ patients with measurable CRP levels were ultimately included in this analysis. The CRP value at the time of diagnosis was used as the index CRP level. The patients were classified into two groups by their index CRP level, with a cutoff at $20 \mathrm{mg} / \mathrm{L}: 373 \mathrm{had}$ a CRP $>20$, and $332 \mathrm{had}$ a CRP $\leq 20$ $\mathrm{mg} / \mathrm{L}$. The patients were 7 to 77 years of age at the time of CD

Table 1. Baseline Characteristics of Patients with Crohn's Disease according to the C-Reactive Protein Level at Diagnosis

\begin{tabular}{|c|c|c|c|}
\hline Variable & $\begin{array}{c}\text { CRP > 20 } \\
(\mathrm{n}=373)\end{array}$ & $\begin{array}{l}C R P \leq 20 \\
(n=332)\end{array}$ & p-value \\
\hline Age, yr & $25.4 \pm 10.1$ & $28.6 \pm 14.2$ & $<0.001$ \\
\hline Sex, male/female & $285 / 88$ & $238 / 94$ & 0.153 \\
\hline Time to follow-up, mo & $86.4 \pm 44.1$ & $83.5 \pm 43.9$ & 0.379 \\
\hline BMI, kg/m² & $19.1 \pm 3.3$ & $20.9 \pm 4.2$ & $<0.001$ \\
\hline Smoking status & & & 0.020 \\
\hline Never smoker & $245(77.8)$ & $168(70.0)$ & \\
\hline Current smoker & $46(14.6)$ & $36(15.0)$ & \\
\hline Ex-smoker & $16(5.1)$ & $31(12.9)$ & \\
\hline Stop smoking after treatment & $8(2.5)$ & $5(2.1)$ & \\
\hline Family history of IBD & $9(2.4)$ & $7(2.1)$ & 0.447 \\
\hline $\mathrm{Hb}, \mathrm{g} / \mathrm{dL}$ & $11.9 \pm 1.9$ & $12.8 \pm 2.1$ & $<0.001$ \\
\hline Hct, \% & 36.0 & 38.1 & $<0.001$ \\
\hline $\mathrm{ESR}, \mathrm{mm} / \mathrm{hr}$ & $47.5 \pm 29.2$ & $23.6 \pm 21.5$ & $<0.001$ \\
\hline CDAI at diagnosis & $217.5 \pm 110.2$ & $164.6 \pm 89.2$ & $<0.001$ \\
\hline Location at diagnosis* & & & $<0.001$ \\
\hline L1 (ileal) & $51(17.5)$ & $91(32.9)$ & \\
\hline L2 (colonic) & $71(24.4)$ & 48 (17.3) & \\
\hline L3 (ileocolonic) & $164(56.4)$ & $134(48.4)$ & \\
\hline L4 (upper GI) & $5(1.7)$ & $4(1.4)$ & \\
\hline Behavior at diagnosis* & & & 0.503 \\
\hline B1 (inflammatory) & $176(71.3)$ & $193(72.6)$ & \\
\hline B2 (stricturing) & $32(13.0)$ & 39 (14.7) & \\
\hline B3 (penetrating) & $39(15.8)$ & $34(12.8)$ & \\
\hline Steroid use at diagnosis & $154(41.2)$ & $84(25.3)$ & $<0.001$ \\
\hline History of anti-TB medication & $67(18.0)$ & $70(21.1)$ & 0.296 \\
\hline History of perianal disease & $108(29.0)$ & $81(24.4)$ & 0.173 \\
\hline
\end{tabular}

Data are presented as mean \pm SD or number $(\%)$.

CRP, C-reactive protein; BMI, body mass index; IBD, inflammatory bowel disease; Hb, hemoglobin; Hct, hematocrit; ESR, erythrocyte sedimentation rate; CDAI, Crohn's disease activity index; GI, gastrointestinal; TB, tuberculosis.

*Location and behavior at the time of diagnosis were unavailable in 137 and 192 patients, respectively. diagnosis, and the mean follow-up duration was 7.1 years. The demographic, clinical and laboratory details of the two groups are summarized in Table 1. There were no significant differences between the groups in gender, family history of inflammatory bowel disease (IBD), history of perianal disease, or history of taking anti-tuberculosis medications. Body mass index (BMI) was significantly lower in the high CRP group. Patients with high CRP were younger at diagnosis than those with low CRP $(\mathrm{p}<0.001)$ and had lower hemoglobin, higher CDAI, and elevated erythrocyte sedimentation rate (ESR). There was a significant difference in lesion location at time of diagnosis $(\mathrm{p}<0.001)$. Le-

Table 2. Disease Manifestation according to the C-Reactive Protein Level at Diagnosis

\begin{tabular}{|c|c|c|c|}
\hline Variable & $\begin{array}{r}\mathrm{CRP}>20 \\
(\mathrm{n}=373)\end{array}$ & $\begin{array}{l}\mathrm{CRP} \leq 20 \\
(\mathrm{n}=332)\end{array}$ & p-value \\
\hline \multicolumn{4}{|l|}{ Involved location } \\
\hline Colorectum & $322(86.3)$ & $222(66.9)$ & $<0.001$ \\
\hline Jejunum & $49(13.1)$ & $61(18.4)$ & 0.651 \\
\hline Ileum & $310(83.1)$ & $270(81.3)$ & 0.917 \\
\hline Esophagogastroduodenum & $36(9.7)$ & $27(8.1)$ & 0.324 \\
\hline \multicolumn{4}{|l|}{ Endoscopic findings } \\
\hline Longitudinal ulcer & $255(68.4)$ & $148(44.6)$ & $<0.001$ \\
\hline Cobblestone appearance & $160(42.9)$ & $57(17.2)$ & $<0.001$ \\
\hline \multicolumn{4}{|l|}{ Histological finding } \\
\hline Granuloma & 148 (39.7) & $110(33.1)$ & 0.657 \\
\hline Stricture & & & 0.027 \\
\hline Yes, at diagnosis & 49 (13.5) & $33(10.0)$ & \\
\hline Yes, during disease course & 46 (12.7) & $29(8.8)$ & \\
\hline Perianal fistula & & & 0.137 \\
\hline Yes, at diagnosis & 105 (28.6) & 77 (23.3) & \\
\hline Yes, during disease course & $35(9.5)$ & $28(8.5)$ & \\
\hline Other fistula & & & 0.145 \\
\hline Yes, at diagnosis & $27(7.4)$ & $10(3.1)$ & \\
\hline Yes, during disease course & $28(7.7)$ & $22(6.7)$ & \\
\hline Abdominal abscess & & & 0.068 \\
\hline Yes, at diagnosis & $20(5.5)$ & $14(4.3)$ & \\
\hline Yes, during disease course & $31(8.5)$ & $17(5.3)$ & \\
\hline Perforation & & & 0.103 \\
\hline Yes, at diagnosis & $14(3.8)$ & $14(4.3)$ & \\
\hline Yes, during disease course & $6(1.6)$ & $12(3.7)$ & \\
\hline Intestinal cancer* & & & 0.659 \\
\hline Yes, at diagnosis & $1(0.3)$ & $3(0.9)$ & \\
\hline Yes, during disease course & $2(0.5)$ & 0 & \\
\hline Primary sclerosing cholangitis & & & 0.472 \\
\hline No & $364(100)$ & $324(99.7)$ & \\
\hline Yes, during disease course & 0 & $1(0.3)$ & \\
\hline
\end{tabular}

Data are presented as number (\%).

CRP, C-reactive protein.

*Includes small bowel and colon cancer. 
sions in the high CRP group more frequently involved the colon (24.4\%) and ileocolon (56.4\%), while the low CRP group had more frequent terminal ileal (32.9\%) and ileocolonic (48.4\%) involvement. The CRP level did not distinguish disease behavior ( $p=0.503$ ); the nonstricturing and nonpenetrating phenotype (B1) was the most frequent disease behavior type in both groups. Location and behavior at diagnosis were unavailable in 137 and 192 patients, respectively. Corticosteroid use at the time of diagnosis was significantly higher in the high CRP group compared with the low CRP group (41.2\% vs 25.3\%, p<0.001).

\section{Disease manifestation}

Colorectal involvement during the course of the disease was more frequent in the high CRP group $(\mathrm{p}<0.001)$. There were no statistically significant differences in jejunal, ileal, and esophagogastroduodenal involvement between the two groups. Endoscopic findings of longitudinal ulcer and cobblestone appearance were more frequent in the high CRP group $(\mathrm{p}<0.001$ and $\mathrm{p}<0.001$, respectively). Stricture during the course of the disease was more frequent in the high compared to the low CRP group $(p=0.027)$. There were no significant differences in the development of fistulas, intestinal perforations, abscesses, intestinal
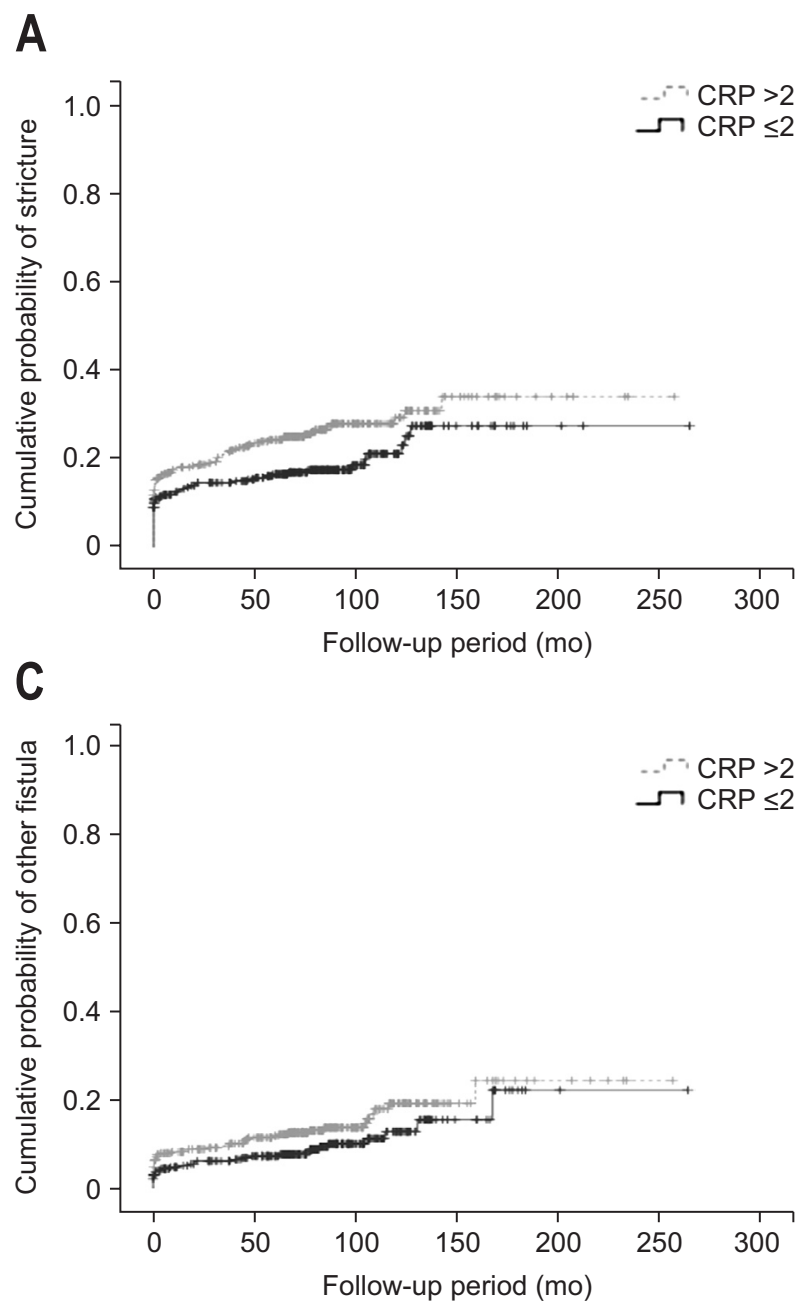

cancers, or primary sclerosing cholangitis between the groups (Table 2). Using the Kaplan-Meier method, significant difference was found in the cumulative probability of stricture according to the CRP level at diagnosis ( $p=0.019)$. However, cumulative probability of perianal fistula and other fistula did not differ (Fig. 1).

\section{Use of medications}

Medication use was investigated according to the index CRP level (Table 3). There were significant differences between the two groups in the use of 5-ASA-based agents, antibiotics, corticosteroids, azathioprine, and biological agents $(\mathrm{p}<0.001$, $\mathrm{p}<0.001, \mathrm{p}<0.001, \mathrm{p}<0.001$, and $\mathrm{p}=0.042$, respectively). Of these medications, more subjects with high CRP had used infliximab $(p=0.023)$, but there was no significant difference in adalimumab use between the two groups. The cumulative use of azathioprine and TNF blocker was significantly higher in high CRP group than in low CRP group (Fig. 2).

\section{Hospital admission, surgery, and survival}

The total numbers of hospital admissions and readmissions were also analyzed according to the index CRP level. The total

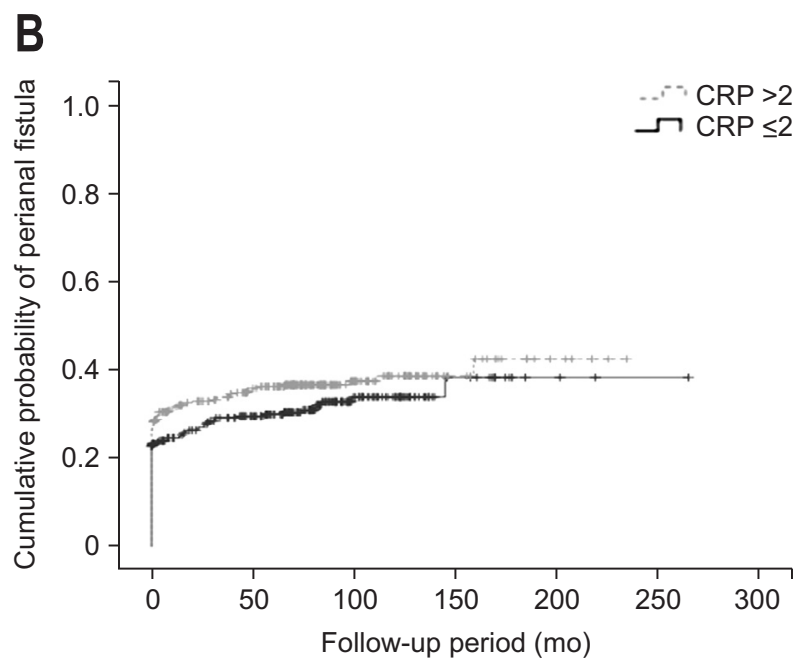

Fig. 1. (A) Kaplan-Meier curve showing the cumulative probability of stricture according to the C-reactive protein (CRP) level (log-rank test, $\mathrm{p}=0.019$ ). (B) Kaplan-Meier curve showing the cumulative probability of perianal fistula according to the CRP level (log-rank test, $p=0.157$ ). (C) Kaplan-Meier curve showing the cumulative probability of other fistula according to the CRP level (log-rank test, $\mathrm{p}=0.086$ ). 
Table 3. Use of Medications according to the C-Reactive Protein Level at Diagnosis

\begin{tabular}{lccc}
\hline \multicolumn{1}{c}{ Variable } & $\begin{array}{c}\text { CRP }>20 \\
(\mathrm{n}=373)\end{array}$ & $\begin{array}{c}\text { CRP } \leq 20 \\
(\mathrm{n}=332)\end{array}$ & $\mathrm{p}$-value \\
\hline Use of medications & $368(98.7)$ & $309(93.1)$ & $<0.001$ \\
5-ASA & $239(64.1)$ & $153(46.1)$ & $<0.001$ \\
Antibiotics & $42(11.3)$ & $32(9.6)$ & 0.494 \\
Budesonide & $263(70.5)$ & $164(49.7)$ & $<0.001$ \\
Corticosteroid & $281(75.3)$ & $194(58.4)$ & $<0.001$ \\
Azathioprine & $4(1.1)$ & $6(1.8)$ & 0.407 \\
MTX & $102(27.4)$ & $66(19.9)$ & 0.023 \\
Infliximab & $21(5.6)$ & $15(4.5)$ & 0.516 \\
Adalimumab & $111(29.8)$ & $76(22.9)$ & 0.042 \\
TNF blocker & $77(20.6)$ & $59(17.8)$ & 0.796 \\
TPN & $28.8 \pm 34.0$ & $28.6 \pm 31.3$ & 0.950 \\
Time to use of azathioprine, mo & $52.2 \pm 41.1$ & $61.8 \pm 43.1$ & 0.114 \\
Time to use of TNF blockers, mo & & &
\end{tabular}

Data are presented as number $(\%)$ or mean \pm SD.

CRP, C-reactive protein; ASA, aminosalicylic acid; MTX, methotrexate; TNF, tumor necrosis factor; TPN, total parenteral nutrition.
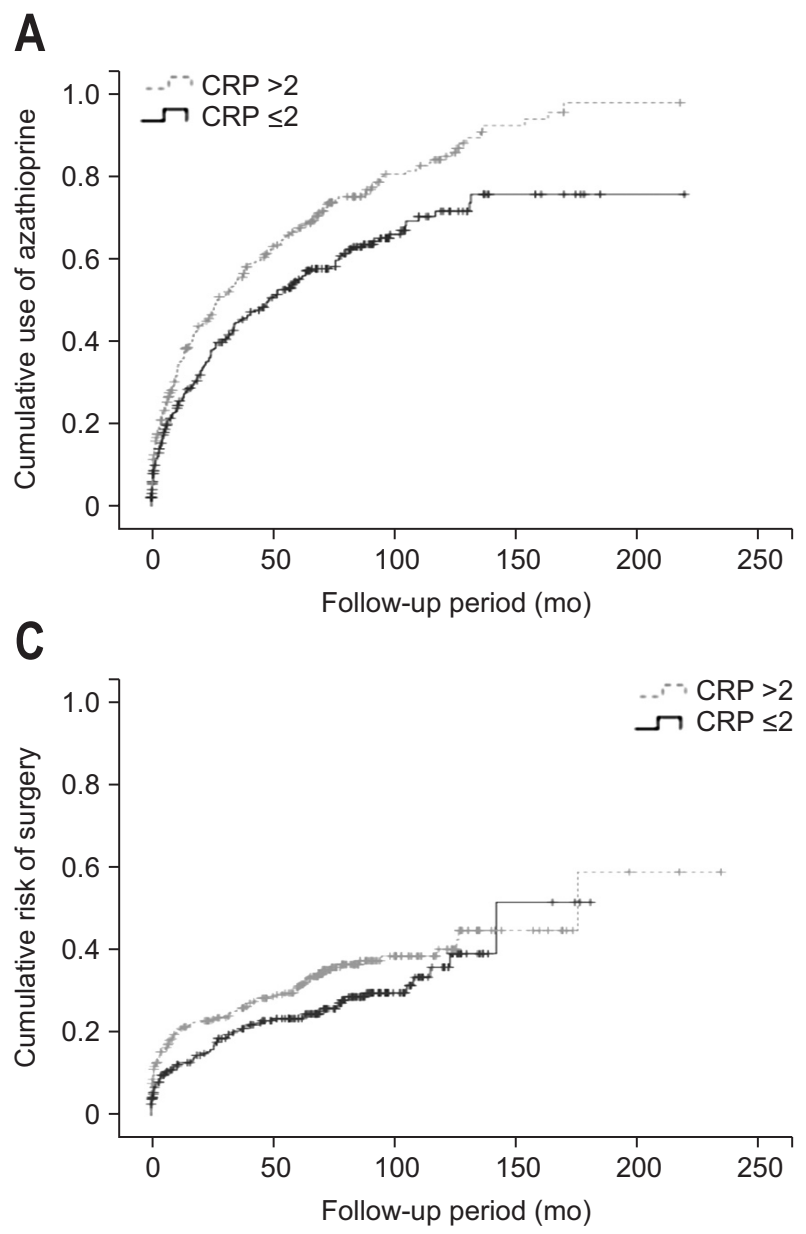

number of hospital admissions differed significantly between the patients with high or low CRP $(3.2 \pm 4.2$ vs $2.3 \pm 3.7, p=0.011)$. In addition, the rate of readmission was higher in the high CRP group ( $\mathrm{p}=0.048)$. Regarding CD-related surgeries, there were no significant differences in the total number of surgeries or the proportion of patients who underwent surgery for intestinal or perianal lesions between the two groups, nor did they differ in the proportion of repeat surgeries for intestinal or perianal lesions. The cumulative risk of surgery and resurgery did not differ according to the CRP level at diagnosis (Fig. 2). Only two patients died during the follow-up period: one from cardiac arrest of unknown cause and the other from intestinal bleeding associated with CD (Table 4).

\section{Predictors of hospitalization}

We performed logistic regression analysis to identify factors that predicted hospitalization (Table 5). In univariate analysis, high index CRP, younger age, use of a TNF blocker, high CDAI at diagnosis, and a stricturing phenotype were all significant risk factors for hospitalization. Variables including high CRP

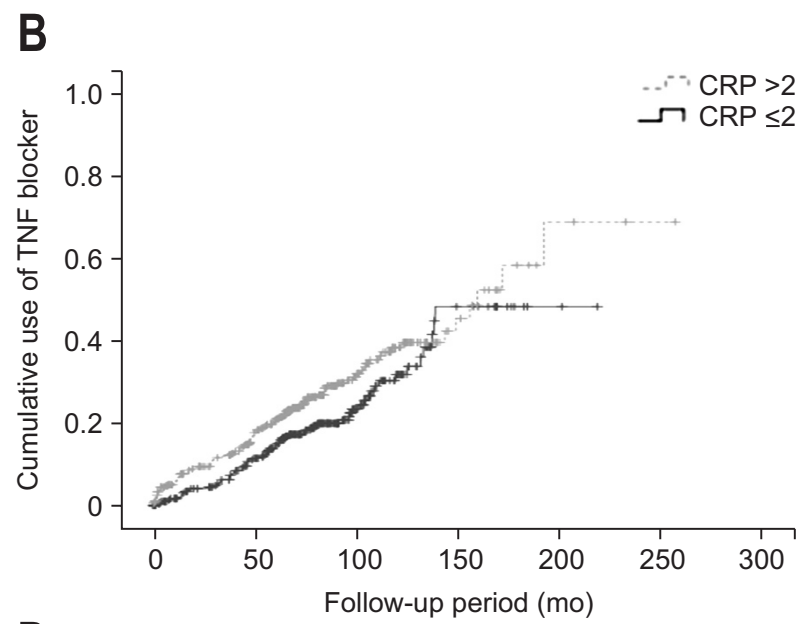

D

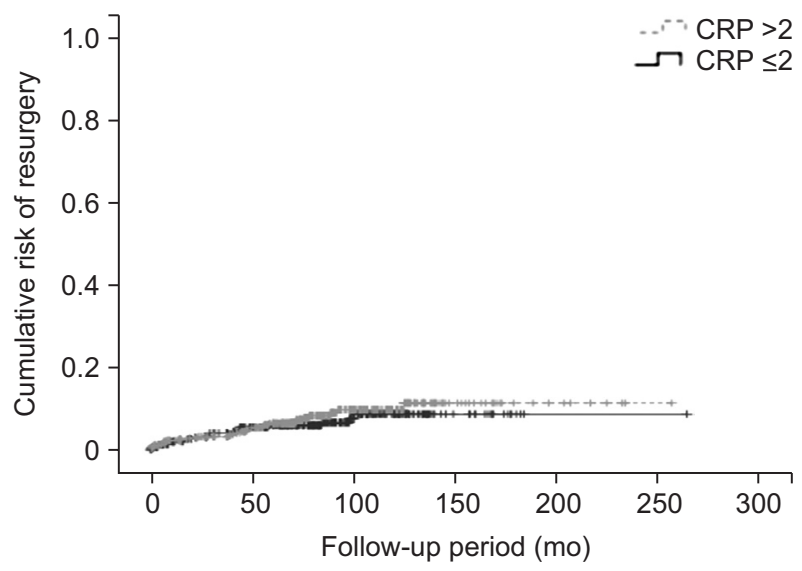

Fig. 2. (A) Kaplan-Meier curve showing the cumulative use of azathioprine according to the C-reactive protein (CRP) level (log-rank test, $p<0.001)$. (B) Kaplan-Meier curve showing the cumulative use of a tumor necrosis factor blocker according to the CRP level (log-rank test, $p=0.041$ ). (C) Kaplan-Meier curve showing cumulative risk of surgery according to the CRP level (log-rank test, $\mathrm{p}=0.067$ ). (D) Kaplan-Meier curve showing cumulative risk of resurgery according to the CRP level (log-rank test, $p=0.489$ ). 
(hazard ratio [HR], 2.03; 95\% confidence interval [CI], 1.10 to 3.82; $\mathrm{p}=0.028$ ), use of a TNF blocker (HR, 22.90; 95\% CI, 3.10 to $172.10 ; \mathrm{p}=0.002$ ), and a stricturing phenotype (HR, 11.31; 95\% CI, 2.62 to $48.81 ; p=0.001$ ) were also significant risk factors for hospitalization in the multivariate analysis.

\section{DISCUSSION}

In this study, CD patients with CRP $>20 \mathrm{mg} / \mathrm{L}$ were more likely to be younger and exhibit colonic or ileocolonic involvement. The CRP level was a useful index of clinical and endoscopic disease activity. During follow-up, high CRP was associated with more aggressive treatments, such as immunosuppressants and biological agents, and with the total number of hospital admissions and readmissions.

CRP was described as a marker for the differential diagnosis of CD and ulcerative colitis, ${ }^{15}$ and the association between CRP and disease activity has been investigated. Several recent studies have reported a correlation between the index CRP and clinical relapses, the subsequent need for aggressive treatment, and response to treatment. ${ }^{19,25}$ However, research supporting the CRP level at the time of diagnosis as a predictor of the clinical course is lacking.

Table 4. Hospital Admission, Surgery, and Survival according to the C-Reactive Protein Level at Diagnosis

\begin{tabular}{lccc}
\hline \multicolumn{1}{c}{ Variable } & $\begin{array}{c}\text { CRP }>20 \\
(\mathrm{n}=373)\end{array}$ & $\begin{array}{c}\text { CRP } \leq 20 \\
(\mathrm{n}=332)\end{array}$ & $\mathrm{p}$-value \\
\hline Total no. of admission & $3.10 \pm 4.20$ & $2.30 \pm 3.70$ & 0.011 \\
Readmission & $193(51.7)$ & $147(44.3)$ & 0.048 \\
Patients performed surgery & $179(48.0)$ & $146(44.0)$ & 0.286 \\
Total no. of surgeries & $1.50 \pm 0.84$ & $1.50 \pm 0.81$ & 0.823 \\
Surgery of intestinal lesion & $82(22.0)$ & $74(22.3)$ & 0.922 \\
Surgery of perianal lesion & $109(29.2)$ & $76(22.9)$ & 0.056 \\
Resurgery of intestinal lesion & $15(4.0)$ & $9(2.7)$ & 0.338 \\
Resurgery of perianal lesion & $26(7.0)$ & $25(7.5)$ & 0.775 \\
Dead & $1(0.3)$ & $1(0.3)$ & 0.932 \\
\hline
\end{tabular}

Data are presented as mean \pm SD or number (\%).

CRP, C-reactive protein.
In a recent study by the Brisbane IBD research group, the low CRP group had a strikingly lower BMI and more pure ileal disease (predisposing them to intestinal resections) than the high CRP group. ${ }^{26}$ A retrospective study in Germany also reported disease with an exclusively ileal distribution in the low CRP group. ${ }^{27}$ Although we could not reproduce the correlation between a low BMI and prior intestinal resection, we confirmed that patients with low CRP tend to develop ileal disease, while those with high CRP have a higher frequency of colonic involvement. Our results are consistent with those of previous studies. ${ }^{16,18,28}$ Although the exact mechanism underlying the predominance of ileal lesions in the low CRP group is not understood, a study has indicated that ileal disease has a tendency to evolve more locoregional inflammatory disease, as evidenced by a high rate of fat wrapping in surgical specimens, than systemic disease and, consequently, does not elevate CRP. ${ }^{26}$

Studies of the Montreal classification of $\mathrm{CD}$ behavior based on the CRP level have yielded conflicting results. In a prospective population-based study, noninflammatory behavior including stricturing or penetrating disease was associated with a high CRP at the time of diagnosis. ${ }^{25}$ By contrast, a retrospective database-based study concluded that there was no significant difference in disease behavior based on the index CRP level during a clinical relapse. ${ }^{19}$ However, little information is available regarding changes in disease behavior during the course of the disease among these patients. In our study, there was no significant difference in disease behavior between the two groups at the initial presentation of $\mathrm{CD}$, and inflammatory disease was the predominant phenotype in both groups. In addition, we found that intestinal strictures occurred more frequently in high CRP patients throughout the disease course. Our findings suggest that high CRP patients have a higher likelihood of fibrostenotic changes because they have a chronic inflammatory profile. We therefore believe that CRP can be a valuable tool to predict further stricture formation, which is a major indication for surgery.

We also observed that high CRP was significantly correlated with a cobblestone appearance and longitudinal ulcers at colonoscopy, which suggest active inflammation. In addition, the CDAI score of high CRP patients was higher than the score of those with low CRP. These results correspond with those of a

Table 5. Multivariate Logistic Regression Analysis to Evaluate Potential Predictors for Hospitalization

\begin{tabular}{|c|c|c|c|c|c|c|}
\hline \multirow{2}{*}{ Variable } & \multicolumn{3}{|c|}{ Univariate analysis } & \multicolumn{3}{|c|}{ Multivariate analysis } \\
\hline & HR & $95 \% \mathrm{CI}$ & p-value & $\mathrm{HR}$ & $95 \% \mathrm{CI}$ & p-value \\
\hline $\mathrm{CRP},>20 \mathrm{mg} / \mathrm{L}$ & 2.30 & $1.63-3.26$ & $<0.001$ & 2.03 & $1.10-3.82$ & 0.028 \\
\hline Age & 0.98 & $0.97-0.99$ & 0.004 & 0.99 & $0.97-1.02$ & 0.761 \\
\hline Use of TNF blocker & 7.16 & $3.88-13.23$ & $<0.001$ & 22.90 & $3.10-172.10$ & 0.002 \\
\hline CDAI at diagnosis & 1.00 & $1.00-1.01$ & 0.021 & 1.00 & $1.00-1.01$ & 0.255 \\
\hline Stricturing phenotype & 3.86 & $2.23-6.69$ & $<0.001$ & 11.31 & $2.62-48.81$ & 0.001 \\
\hline
\end{tabular}

HR, hazard ratio; CI, confidence interval; CRP, C-reactive protein; TNF, tumor necrosis factor; CDAI, Crohn's disease activity index. 
retrospective study of 147 American patients that demonstrated that an elevated CRP was associated with clinical disease activity, endoscopic inflammation, and several other IBD biomarkers. $^{16}$

CRP can be used to predict subsequent need for azathioprine and biological agents as well as clinical relapse. ${ }^{19}$ A retrospective study in the Netherlands provided additional evidence for the role of CRP in predicting a more severe clinical course of $\mathrm{CD}$, such as number of relapses, severity of relapse, and cumulative days of prednisone use. ${ }^{25}$ In accord with previous studies, we determined that patients with high CRP received more aggressive medical treatment, such as immunosuppressants or biological agents. Our multivariate analysis also indicated that a high CRP level is an independent predictor for hospitalization. Interestingly, the CRP groups did not differ in their need for surgery or repeat surgery. These results coincide well with those of a previous study that reported that patients with low CRP more often have purely ileal disease, with an increased requirement for intestinal resection. ${ }^{26}$ Accordingly, we can postulate that the surgery rate in patients with low CRP would be similar to that of those with a high CRP, in whom more aggressive treatment would be required.

In the present study, we observed a correlation of CRP levels with clinical progression to severe disease. Although the precise mechanisms underlying this correlation have not been fully elucidated, some plausible explanation for these results can be inferred from some published reports. Mesenteric fat, rather than the intestinal wall, is likely important for the production of CRP in CD patients. ${ }^{29}$ The mesenteric fat hyperplasia observed in $\mathrm{CD}$ but not UC has been suggested to contribute to the synthesis of inflammatory mediators such as TNF- $\alpha$ and IL-6, which promote damage to the intestinal mucosa. In addition, mesenteric fat hyperplasia could impair gut barrier function by disturbing innate immunity to the gut flora. ${ }^{30}$ Based on these findings, the elevation of CRP at baseline could reflect the degree of mesenteric fat hyperplasia. Accordingly, increased production of inflammatory cytokines via the accumulation of mesenteric adipocytes might induce a severe, ongoing, inflammatory process, and direct assessment of this inflammatory activity could facilitate the prediction of a more severe clinical course.

Several studies have presented different CRP cutoff values for CD patients, ${ }^{19,25,31}$ but a definitive cutoff value for predicting disease activity and clinical course has not been established. In a study of French patients, high CRP ( $>20 \mathrm{mg} / \mathrm{L}$ ) was emphasized as a valuable diagnostic marker for predicting a van Hees index $\geq 150$. $^{32}$ The GETAID group emphasized that CRP $>20 \mathrm{mg} / \mathrm{L}$ and ESR $>15 \mathrm{~mm} / \mathrm{hr}$ were the only meaningful laboratory markers predictive of relapse. ${ }^{31}$ Furthermore, a multicenter randomized controlled study indicated that high CRP ( $>20 \mathrm{mg} / \mathrm{L}$ ) was correlated with relapse after azathioprine withdrawal. ${ }^{33}$ These results determined our selection of $20 \mathrm{mg} / \mathrm{L}$ as the optimal CRP cutoff value for categorizing the disease manifestations and clinical course of CD.

This study improves upon previous studies by employing a multicenter cohort from 32 institutions of CD patients with measurable CRP. This design minimizes bias that might arise from a single center case-control study. We also enrolled a significant proportion of potential cases, ultimately including 705 patients and thereby substantially increasing the statistical power. Furthermore, uniform findings based on the CRP level were reproduced in this study despite the heterogeneity of the studied population. The classification of disease phenotype, activity and clinical course by CRP level may therefore be applicable to the patients with $\mathrm{CD}$ in clinical practice.

Our study is limited by its retrospective nature, which limits our ability to provide a causal explanation for the relationship between CRP levels and clinical outcomes.

This study includes missing values on potentially important covariates, which could be unavoidable in retrospective and observational study. However, unavailable data is mostly due to insufficient data collection and work-up or loss to followup before electronic medical record was introduced in the early 2000s. In addition, there is a possibility of poor quality assurance concerning admission, treatment, and follow-up and patient enrollment with relatively severe disease state due to characteristics of participating secondary or tertiary care center, which could limit reliability of the study. However, these potential confounders were minimized by the retrieval of clinical data from electronic medical record systems at all participating hospitals and accurate recording of the time to occurrence of any event or use of medication. Unfortunately, we could not investigate clinical data on the frequency or severity of relapses, improvement of subjective symptoms, and treatment response, particularly for biological agents, due to the lack of medical records and the observational study design. In conclusion, CRP at the time of diagnosis appears to be useful in predicting disease phenotype, clinical and endoscopic activity, and the clinical course of CD. Early aggressive treatment with intensive followup management strategy may be warranted in patients with high CRP. In addition, the integration of CRP levels with other predictive indices will improve the reliability of the identification of the natural history of $\mathrm{CD}$. A further prospective study that includes a large number of subjects could provide definitive evidence of the correlation of CRP levels with multiple clinical outcomes.

\section{CONFLICTS OF INTEREST}

No potential conflict of interest relevant to this article was reported.

\section{ACKNOWLEDGEMENTS}

This study was supported by the Research Program funded 
by the Korea Centers for Disease Control and Prevention (2013E63004-01).

\section{REFERENCES}

1. Mazor Y, Maza I, Kaufman E, et al. Prediction of disease complication occurrence in Crohn's disease using phenotype and genotype parameters at diagnosis. J Crohns Colitis 2011;5:592-597.

2. Jung YS, Song CS, Kim ER, et al. Seasonal variation in months of birth and symptom flares in Korean patients with inflammatory bowel disease. Gut Liver 2013;7:661-667.

3. Chang CW, Wong JM, Tung CC, Shih IL, Wang HY, Wei SC. Intestinal stricture in Crohn's disease. Intest Res 2015;13:19-26.

4. Hu PJ. Inflammatory bowel disease in Asia: the challenges and opportunities. Intest Res 2015;13:188-190.

5. Kotze PG, Spinelli A, da Silva RN, et al. Conventional versus biological therapy for prevention of postoperative endoscopic recurrence in patients with Crohn's disease: an International, multicenter, and observational study. Intest Res 2015;13:259-265.

6. D’Incà R, Dal Pont E, Di Leo V, et al. Can calprotectin predict relapse risk in inflammatory bowel disease? Am J Gastroenterol 2008;103:2007-2014.

7. Cotterill L, Payne D, Levinson S, et al. Replication and meta-analysis of 13,000 cases defines the risk for interleukin-23 receptor and autophagy-related 16-like 1 variants in Crohn's disease. Can J Gastroenterol 2010;24:297-302.

8. Lichtenstein GR. Emerging prognostic markers to determine Crohn's disease natural history and improve management strategies: a review of recent literature. Gastroenterol Hepatol (NY) 2010;6:99-107.

9. Van Limbergen J, Russell RK, Nimmo ER, Satsangi J. The genetics of inflammatory bowel disease. Am J Gastroenterol 2007;102: 2820-2831.

10. Lee KM. Fecal biomarkers in inflammatory bowel disease. Intest Res 2013;11:73-78.

11. Vermeire S, Van Assche G, Rutgeerts P. C-reactive protein as a marker for inflammatory bowel disease. Inflamm Bowel Dis 2004; 10:661-665.

12. Jürgens M, Mahachie John JM, Cleynen I, et al. Levels of Creactive protein are associated with response to infliximab therapy in patients with Crohn's disease. Clin Gastroenterol Hepatol 2011;9:421-427.e1.

13. Mendall MA, Strachan DP, Butland BK, et al. C-reactive protein: relation to total mortality, cardiovascular mortality and cardiovascular risk factors in men. Eur Heart J 2000;21:1584-1590.

14. Imhof A, Froehlich M, Brenner H, Boeing H, Pepys MB, Koenig W. Effect of alcohol consumption on systemic markers of inflammation. Lancet 2001;357:763-767.

15. Vermeire S, Van Assche G, Rutgeerts P. Laboratory markers in IBD: useful, magic, or unnecessary toys? Gut 2006;55:426-431.

16. Solem CA, Loftus EV Jr, Tremaine WJ, Harmsen WS, Zinsmeister AR, Sandborn WJ. Correlation of C-reactive protein with clinical, endoscopic, histologic, and radiographic activity in inflammatory bowel disease. Inflamm Bowel Dis 2005;11:707-712.

17. Cellier C, Sahmoud T, Froguel E, et al. Correlations between clinical activity, endoscopic severity, and biological parameters in colonic or ileocolonic Crohn's disease: a prospective multicentre study of 121 cases. The Groupe d'Etudes Thérapeutiques des Affections Inflammatoires Digestives. Gut 1994;35:231-235.

18. Lakatos PL, Kiss LS, Palatka K, et al. Serum lipopolysaccharidebinding protein and soluble CD14 are markers of disease activity in patients with Crohn's disease. Inflamm Bowel Dis 2011;17:767777.

19. Koelewijn CL, Schwartz MP, Samsom M, Oldenburg B. C-reactive protein levels during a relapse of Crohn's disease are associated with the clinical course of the disease. World J Gastroenterol 2008; 14:85-89.

20. Magro F, Rodrigues-Pinto E, Santos-Antunes J, et al. High Creactive protein in Crohn's disease patients predicts nonresponse to infliximab treatment. J Crohns Colitis 2014;8:129-136.

21. Cheon JH, Kim YS, Ye BD, et al. Crohn's Disease Clinical Network and Cohort (CONNECT) Study: the first step toward nationwide multicenter research of Crohn's disease in Korea. Intest Res 2014;12:173-175.

22. Lee JW, Im JP, Cheon JH, Kim YS, Kim JS, Han DS. Inflammatory bowel disease cohort studies in Korea: present and future. Intest Res 2015;13:213-218.

23. Carter MJ, Lobo AJ, Travis SP; IBD Section, British Society of Gastroenterology. Guidelines for the management of inflammatory bowel disease in adults. Gut 2004;53 Suppl 5:V1-V16.

24. Bernstein CN, Loftus EV, Ng SC, Lakatos PL, Moum B. Hospitalisations and surgery in Crohn's disease. Gut 2012;61:622-629.

25. Kiss LS, Papp M, Lovasz BD, et al. High-sensitivity C-reactive protein for identification of disease phenotype, active disease, and clinical relapses in Crohn's disease: a marker for patient classification? Inflamm Bowel Dis 2012;18:1647-1654.

26. Florin TH, Paterson EW, Fowler EV, Radford-Smith GL. Clinically active Crohn's disease in the presence of a low C-reactive protein. Scand J Gastroenterol 2006;41:306-311.

27. Thalmaier D, Dambacher J, Seiderer J, et al. The $+1059 \mathrm{G} / \mathrm{C}$ polymorphism in the C-reactive protein (CRP) gene is associated with involvement of the terminal ileum and decreased serum CRP levels in patients with Crohn's disease. Aliment Pharmacol Ther 2006;24:1105-1115.

28. Yang DH, Yang SK, Park SH, et al. Usefulness of C-reactive protein as a disease activity marker in Crohn's disease according to the location of disease. Gut Liver 2015;9:80-86.

29. Peyrin-Biroulet L, Gonzalez F, Dubuquoy L, et al. Mesenteric fat as a source of $\mathrm{C}$ reactive protein and as a target for bacterial translocation in Crohn's disease. Gut 2012;61:78-85

30. Peyrin-Biroulet L, Chamaillard M, Gonzalez F, et al. Mesenteric fat in Crohn's disease: a pathogenetic hallmark or an innocent bystander? Gut 2007;56:577-583.

31. Consigny Y, Modigliani R, Colombel JF, et al. A simple biological 
score for predicting low risk of short-term relapse in Crohn's disease. Inflamm Bowel Dis 2006;12:551-557.

32. Chamouard P, Richert Z, Meyer N, Rahmi G, Baumann R. Diagnostic value of C-reactive protein for predicting activity level of
Crohn's disease. Clin Gastroenterol Hepatol 2006;4:882-887.

33. Treton X, Bouhnik Y, Mary JY, et al. Azathioprine withdrawal in patients with Crohn's disease maintained on prolonged remission: a high risk of relapse. Clin Gastroenterol Hepatol 2009;7:80-85. 